\title{
OBSERVATION OF DELAYED PROTON RADIOACTIVITY
}

R. Barton, R. McPherson, R E. Bell, W. R. Frisken, W. T. LINK, AND R. B. MOORE 


\title{
OBSERVATION OF DELAYED PROTON RADIOACTIVITY
}

\author{
R. Barton, ${ }^{*}$ R. McPherson, R. E. Bell, W. R. Frisken, \\ W. T. Link, AND R. B. MoORE \\ Radiation Laboratory, McGill University, Montreal \\ Received August 14, 1963
}

\begin{abstract}
The observation of delayed proton emission is reported. Bombardment of both aluminum and silicon with $97-$ Mev protons leads to the emission of delayed proton energy groups from 2 to $5 \mathrm{Mev}$. They follow the beta decay of $\mathrm{Si}^{25}\left(T_{1 / 2}\right.$ about $0.3 \mathrm{sec}$ ) to proton-unstable excited states of $\mathrm{Al}^{25}$. Delayed protons were also observed following bombardment of $\mathrm{Mg}, \mathrm{Na}, \mathrm{F}, \mathrm{N}$, and $\mathrm{O}$. The energy groups in the delayed proton spectra are explained by assuming that, in each case, a nuclide whose $(Z, N)$ is of the form $(2 k+2,2 k-1)$ is the parent nuclide, the decay chain being of the form:

$$
(2 k+2,2 k-1) \stackrel{\beta^{+}}{\rightarrow}(2 k+1,2 k) \stackrel{p}{\rightarrow}(2 k, 2 k) .
$$

The observed delayed proton energies are those deduced by assuming that known levels of the intermediate nucleus emit the protons. Agreement of this kind, together with cross bombardment, threshold, and lifetime measurements, established the observation of the previously unreported $\mathrm{Si}^{25}$. With less experimental detail, delayed protons have been assigned to the decay schemes of the previously unreported $\mathrm{Mg}^{21}, \mathrm{Ne}^{17}$, and $\mathrm{O}^{13}$.
\end{abstract}

\section{INTRODUCTION}

Energy groups of delayed protons at 2 to $5 . \mathrm{Mev}$ have been observed following bombardment of Si, Al, $\mathrm{Mg}, \mathrm{Na}, \mathrm{F}, \mathrm{N}$, and $\mathrm{O}$ with $97-\mathrm{Mev}$ protons in the new external beam of the McGill Synchrocyclotron. Only preliminary results are reported, which were obtained just before a planned shutdown of the cyclotron for construction purposes. This work will be resumed shortly. Brief reports of the central part of this work have been made by Barton and McPherson $(1963 a, b)$ and McPherson and Barton (1963). This paper is adapted from the Ph.D. thesis of Barton (1963).

In general, there seem to be three mechanisms through which proton emission that is delayed by a measurable time could occur. (In this experiment all recorded events were delayed by at least $200 \mu \mathrm{sec}$.) The three phenomena will be referred to as: (1) self-delayed proton emission, (2) self-delayed double proton emission, and (3) beta-delayed proton emission. Goldansky (1960, 1961) has discussed these possibilities in some detail. Item (1), self-delayed proton emission, refers to the emission of a low-energy proton delayed by a measurable time owing to the presence of the nuclear Coulomb barrier. (In this language, ordinary $\alpha$ decay would be called self-delayed $\alpha$ emission.) Simple barrier penetration calculations lead to the conclusion that for $Z$ from 10 to 15 , energies about $100 \mathrm{kev}$ may be low enough. Item (2), self-delayed double proton emission, was originally proposed by Goldansky. He predicts that near the edge of proton instability, the binding energy of an even- $Z$ nuclide may be smaller than the energy by which the odd- $Z$ nuclide one proton lighter is

*Present address: Physics Department, Loyola College, Montreal. 
unbound. Thus the heavier nuclide is stable against one-proton decay but unstable against the emission of two protons. Whether one assumes that two individual protons must both penetrate the Coulomb barrier almost simultaneously or that a pair of protons (intranuclear diproton) with its doubled charge and mass must penetrate the Coulomb barrier, simple calculations lead to identical measurable lifetimes. Item (3), beta-delayed proton emission, refers to the branching of the beta decay of a nuclide with large $Q_{\beta}+$ to a protonunstable excited state of the daughter nuclide. In this case the protons would exhibit the lifetime of the beta decay. Goldansky lists nuclides from which each of the above phenomena are to be expected, along with predictions of lifetimes and proton energies.

In this work we have observed beta-delayed proton emission. This is completely analogous to beta-delayed neutron emission, which was first observed by Roberts et al. (1939) and Booth et al. (1939). Pappas and Rudstam (1960) discuss the systematics of delayed neutron emission.

Of the three possible types of delayed proton emitters, one can expect, with a $97-\mathrm{Mev}$ proton beam, to produce beta-delayed proton emitters with considerable probability. On the other hand, the self-delayed $p$ and $2 p$ emitters are at the edge of proton instability, sufficiently far from the stable nuclides so that with such a beam one does not expect to produce them with observable intensity.

\section{EXPERIMENTAL ARRANGEMENTS}

\section{External Beam}

The proton beam used in these bombardments was extracted from the maximum-energy orbit of the McGill Synchrocyclotron by the d-c. magnetic regenerative technique (Moore 1962; Le Couteur 1955). The design of this extraction system and the character of the beam after transport into the new Radiation Laboratory beam hall will be published later. In Fig. 1 the section in the median plane of the cyclotron shows the configuration of the regenerator and extraction channel, together with the simple system used to transport the external proton beam to the temporary experimental station. The self-excited quadrupole sections of the extraction channel were carefully adjusted to render the proton beam optimally parallel as it left the region of strong fringing field. Thus even the weak focusing of the steering magnet was enough to give an average beam intensity of $3 \times 10^{10}$ protons/second through an area of $2 \mathrm{~cm}^{2}$ at the target station, $10 \mathrm{~m}$ from the cyclotron. The geometrical spread of the beam is smaller than $5 \mathrm{~cm}$-milliradians both vertically and horizontally.

The mean proton energy, determined from the extrapolated range in aluminum, is $97.0 \pm 0.3 \mathrm{Mev}$, and preliminary measurements of the energy spread indicate that the FWHM is considerably less than $1 \mathrm{Mev}$.

\section{Apparatus}

Figure 2 gives a representation of the main features of the target chamber. It was evacuated to avoid counting radiations from air and to allow the counting of protons of as low an energy as possible; its vacuum was made separate 


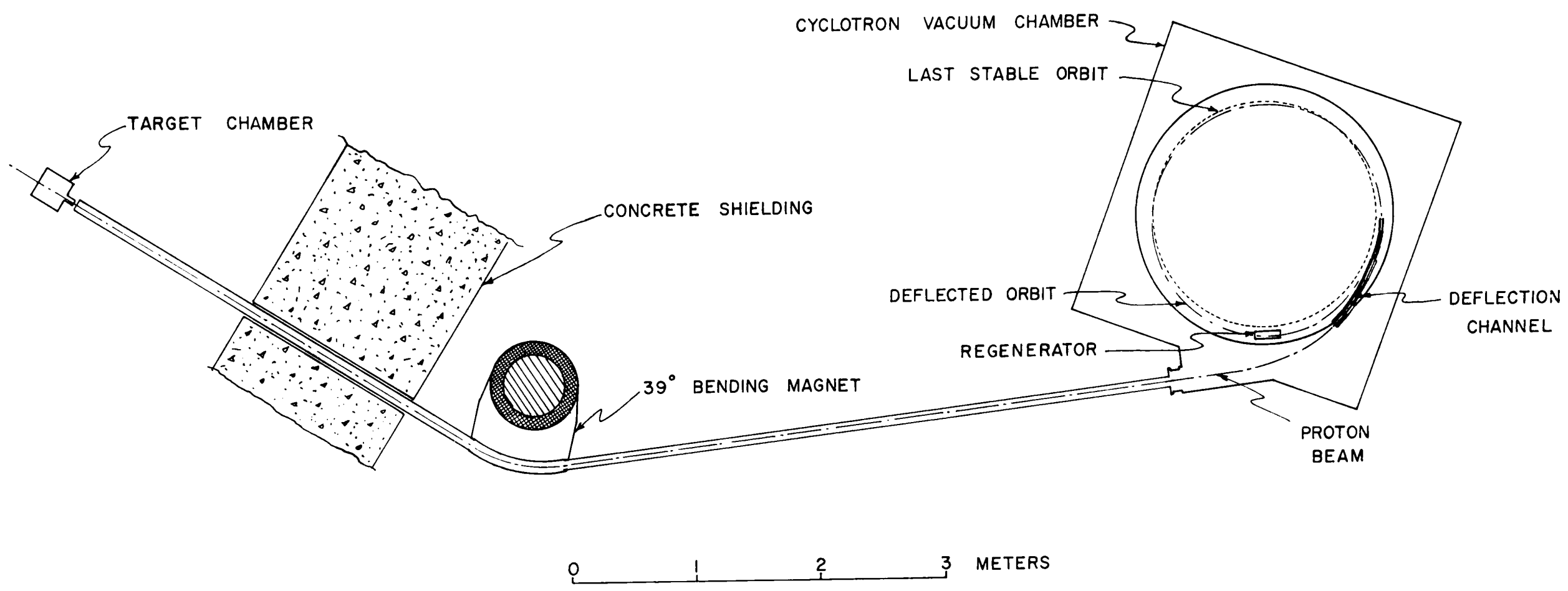

FIG 1. Section in the median plane of the cyclotron, including extraction system, beam transport system, shielding, and position of target chamber. 


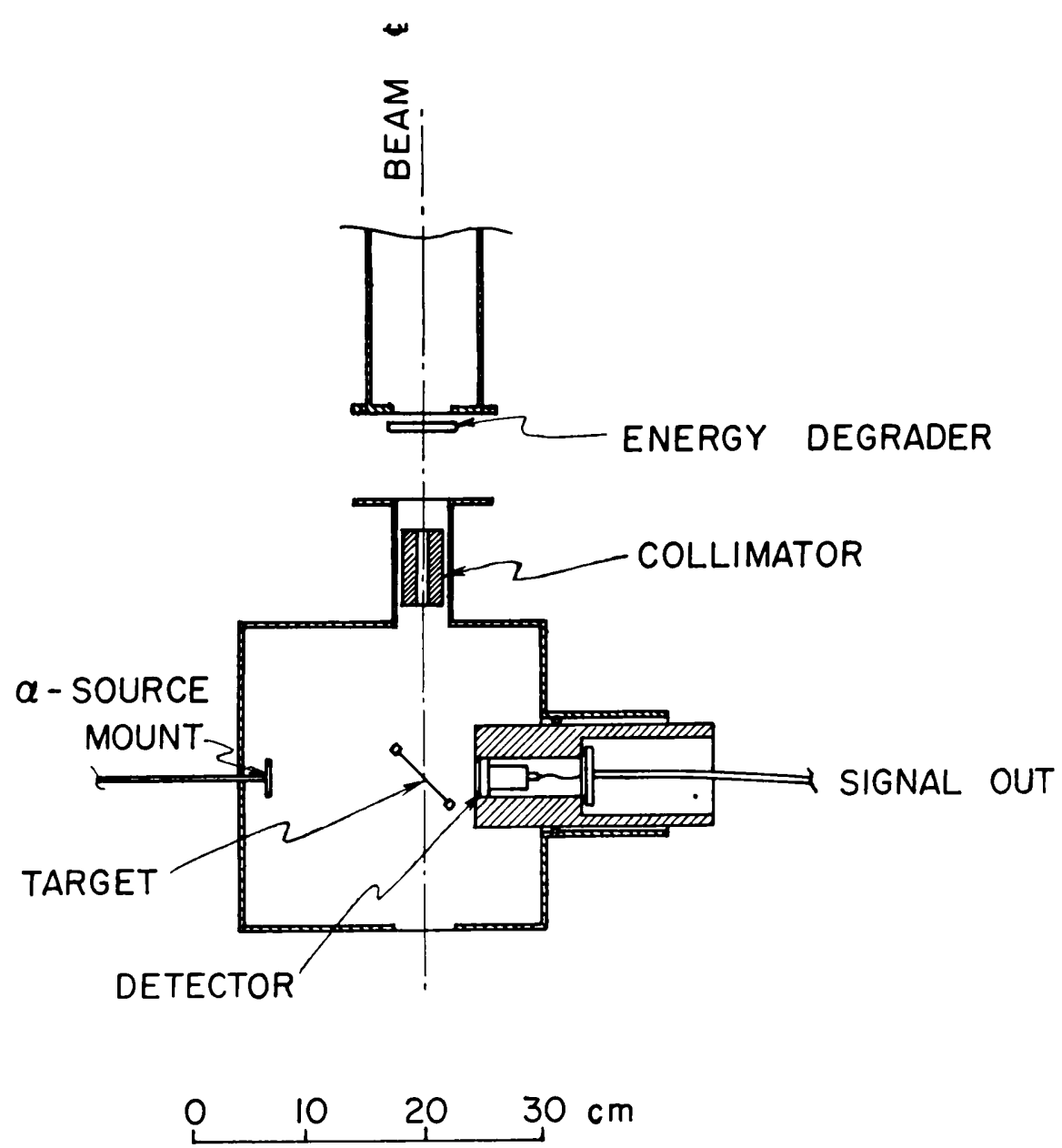

FIG. 2. Schematic representation of the target chamber. The indicated detector is a siliconjunction particle detector. The energy degrader and collimator were used only when obtaining activation curves.

from that of the beam pipe for convenience. The end windows of the chamber were not visible to the counter so that no direct radiations from them were counted. For the purpose of obtaining activation curves, protons of energy lower than $97 \mathrm{Mev}$ were obtained by placing aluminum absorbers of appropriate thickness against the end of the beam pipe. A collimator was then placed as indicated in Fig. 2. In order to calibrate the system for energy response a $\mathrm{Po}^{210} \alpha$ source was mounted on the end of the rod that goes through the chamber wall directly opposite the counter. The source was removed whenever any counting was done.

The counter, a silicon junction particle detector, was protected from the fringe of the beam by the thick-walled aluminum cylinder in which it was mounted. A short piece of coaxial cable $(13 \mathrm{pf} / \mathrm{ft})$ connected the detector to the input of a Tennelec low-noise charge-sensitive amplifier. The face of the counter was $1 \mathrm{in}$. from the axis of the beam. At this distance the effects of scattered protons from each beam burst were such that the electronic system recovered sufficiently for counting to be resumed after $200 \mu \mathrm{sec}$. In order to eliminate delayed- $\alpha$ background and to obtain range-energy curves for the observed energy groups, thin aluminum foils were placed flat on the end of the counter-protecting cylinder. 
The beam current was monitored via a 7 -in. Faraday cup placed a few feet downstream from the exit port of the chamber.

Figure 3 gives a schematic representation of the cyclotron and counting cycles and of the electronic system. The purpose of this apparatus was to store pulse-height information during a selected portion of the interval between beam bursts. The counting interval started $200 \mu \mathrm{sec}$ after each beam

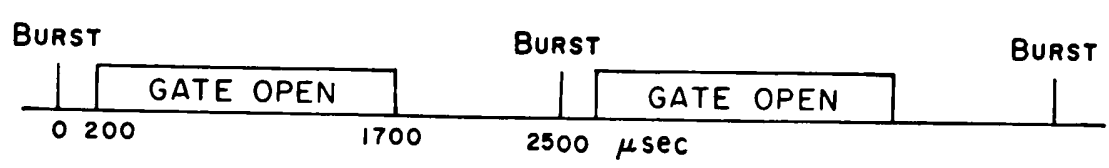

(a)

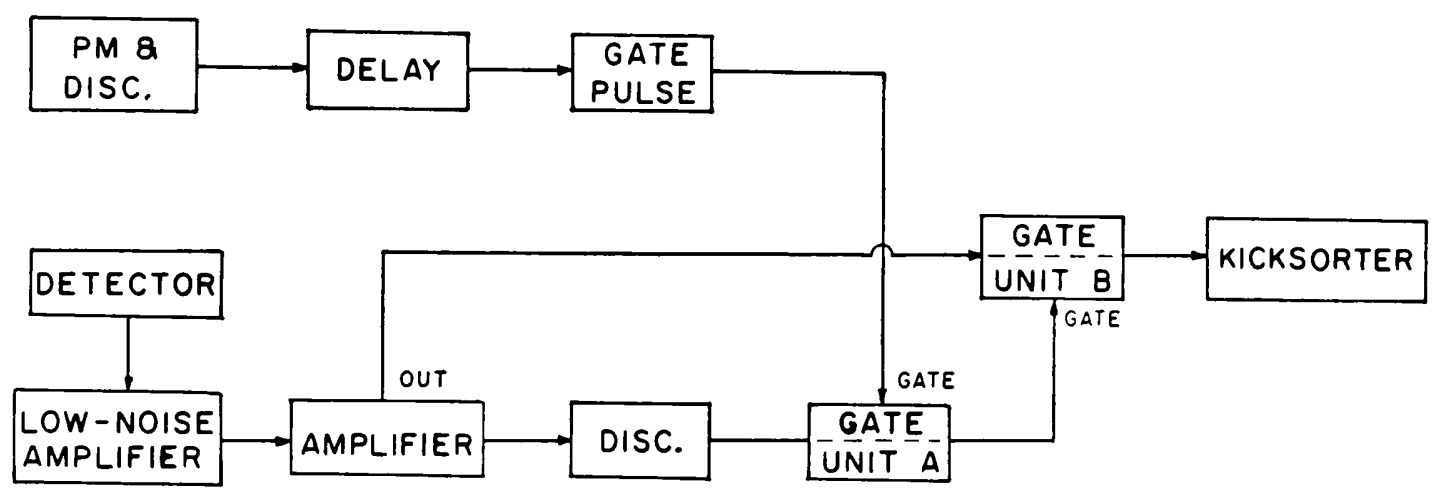

(b)

FIG. 3. (a) Schematic representation of the cyclotron and counting cycles. (b) Block diagram of electronics.

burst and extended to $1700 \mu \mathrm{sec}$ after each burst; if it were any longer it would allow the registering of spurious pulses picked up from the firing-on of the oscillator for the next cyclotron burst. Thus every recorded count was delayed by at least $200 \mu \mathrm{sec}$.

In order to calibrate the system for energy response, i.e., to find the relationship between kicksorter channel and energy absorbed in the detector, the following procedure was used. First a pulse generator giving a long, fastrising pulse was connected to the input of the low-noise amplifier via a 1-pf capacitor. The height of this pulse, and therefore the charge deposited at the amplifier, was precisely controllable by a potentiometer. Using a series of well-known $\alpha$ lines a strict proportionality between $\alpha$ energy and potentiometer setting was established. Therefore after the potentiometer settings were interpreted as energy, the dial being recalibrated from time to time using the $\operatorname{Po}^{210} \alpha$ line.

\section{MEASLREMENTS, RESLLTS, AND DISCUSSION}

In this section the observation of delayed protons following the bombardment of aluminum and the reasons for assigning these to the decay of $\mathrm{Si}^{25}$ will be described in detail. Further, the observation of heavy charged-particle 
energy groups from various other targets will be reported and an explanation of them that is completely analogous to the $\mathrm{Si}^{25}$ explanation for the aluminum target will be given.

\section{Delayed Protons from Aluminum}

Because of very intense background up to about 2.5 Mev due to beta particles and gamma rays, it was not possible to detect any heavy charged particles below this energy. In order to investigate as great an energy range as possible, the RCA F-3-25-2.0 counter, whose rated bias is $25 \mathrm{v}$, was operated regularly at $100 \mathrm{v}$. This seemed to have no effect on the resolution of the system but it did create problems of long-term stability. After some energy groups were found, the detector resistivity was found by plotting the apparent energy of the end of the spectrum against detector bias. The result was a resistivity between 3000 and $5000 \mathrm{ohm}-\mathrm{cm}$. Consequently, with $100-\mathrm{v}$ bias, the thickness of the depletion layer was equal to the range of a proton with energy between 4.5 and $5.0 \mathrm{Mev}$. Thus it was found possible to investigate the energy range from about 2.5 to about $4.5 \mathrm{Mev}$ without distortion or displacement of the peaks. Mainly because of the background conditions, the observed width of monoenergetic peaks was rather large, about $250 \mathrm{kev}$.

Without any target in the counting chamber it was found that during a run of typical length about 20 counts would be distributed over the energy range under investigation. This is completely negligible.

An aluminum foil $2.15 \mathrm{mg} / \mathrm{cm}^{2}$ thick was placed in the target holder (Fig. 2) so that it was in a vertical plane that made an angle of $23^{\circ}$ with the direction of the beam. Thus it was thicker along the direction of the beam than along the line joining it to the counter. The distance from the counter to the beam axis was $2.46 \mathrm{~cm}$, and the counter's area was $200 \mathrm{~mm}^{2}$ so that the geometrical efficiency was about $2.5 \%$. As was previously stated, the delayed gate pulse allowed counts to be recorded during the $1500-\mu \mathrm{sec}$ interval from 200 to 1700 $\mu$ sec after each burst. Since the cyclotron period was held very close to 2500 $\mu \mathrm{sec}$, the counting period occupied $60 \%$ of the time; if the lifetimes of the radiations were long compared to $2500 \mu \mathrm{sec}$, as they seemed to be, this effectively made the counting efficiency $60 \%$ of the geometrical efficiency. With this information, together with the target thickness and beam current, cross sections for the production of delayed protons were estimated.

The delayed spectra following the bombardment of aluminum must be classified into those recorded with a thin or no absorber foil and those recorded with thicker absorbers. With a $4.3 \mathrm{mg} / \mathrm{cm}^{2}$ or thicker $\mathrm{Al}$ foil as absorber the proton peaks stand out quite sharply. With a $2.15-\mathrm{mg} / \mathrm{cm}^{2}$ absorber or with no absorber, the proton spectrum is at least partly buried under a more intense continuum. This continuum is such that $4.3 \mathrm{mg} / \mathrm{cm}^{2}$ of absorber will degrade its uppermost energy from at least $5 \mathrm{Mev}$ to at most $2 \mathrm{Mev}$. This continuum, is therefore, made up of alpha particles because at 5 -Mev energy only an alpha line would be spread into a continuum by a $2.15-\mathrm{mg} / \mathrm{cm}^{2}$-thick source and still get through a $2.15-\mathrm{mg} / \mathrm{cm}^{2}$-thick absorber. It is highly probable that the major source of these alphas is $\mathrm{Na}^{20}$, produced in the reaction $\mathrm{Al}^{27}(p, p \alpha 3 n) \mathrm{Na}^{20}$. This known nuclide has a beta half-life of $0.3 \mathrm{sec}$ and leads to excited states of $\mathrm{Ne}^{20}$ that emit alphas of appropriate energy. 
With the thicker absorbers in place a spectrum consisting of sharp lines is observed. (See Figs. 4 and 5 for examples of such spectra.) These lines unquestionably occur only when the target is in place, but the question remains whether they are delayed radiations from the target or from the counter itself, which is made of silicon. The latter could be the case if, for example,

DELAYED SPECTRUM FROM ALUMINUM TARGET

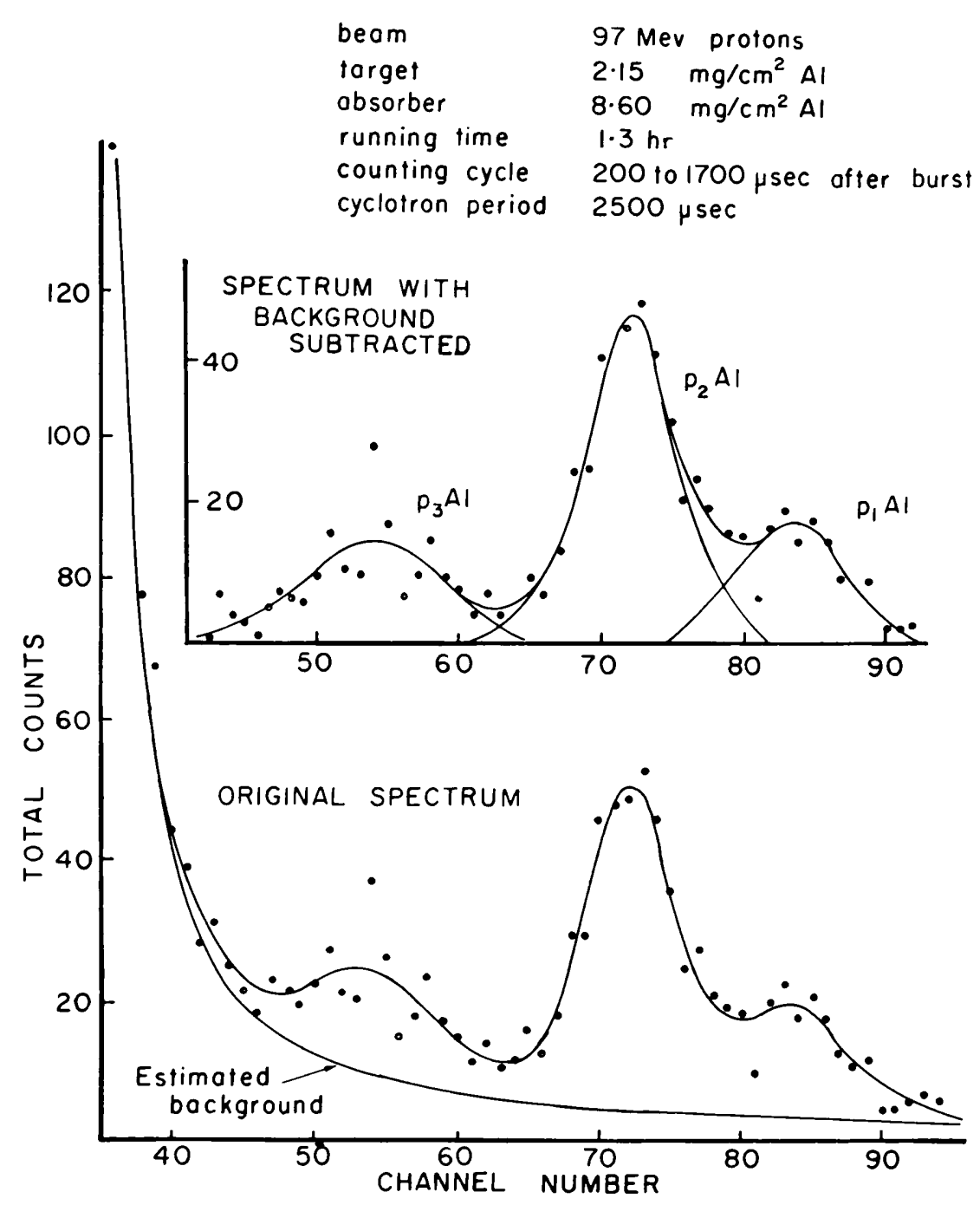

FIG. 4. Spectrum of delayed heavy charged particles from aluminum target. The estimated background was drawn in by hand and subtracted from the original spectrum.

the cross section for production were very much larger in silicon than in aluminum, and protons scattered into the counter by the target initiated the reaction. However, the counted particles must come from the target because, as will be seen later, the qualitative appearance of the spectrum changes when different materials are used in target foils of roughly the same thickness.

Thus energy groups of heavy charged particles that are emitted from the target after a delay of at least $200 \mu \mathrm{sec}$ have been observed. Figure 6 demonstrates that these lines are due to protons. These graphs were obtained from absorption data: various thicknesses of $\mathrm{Al}$ absorber foil were placed over the end of the counter shield and the energy of each peak was determined. The observed energies of the peaks $p_{2} \mathrm{Al}$ and $p_{3} \mathrm{Al}$, as they are labeled in Fig. 4 , are plotted against absorber foil thickness. Residual energy curves are plotted 


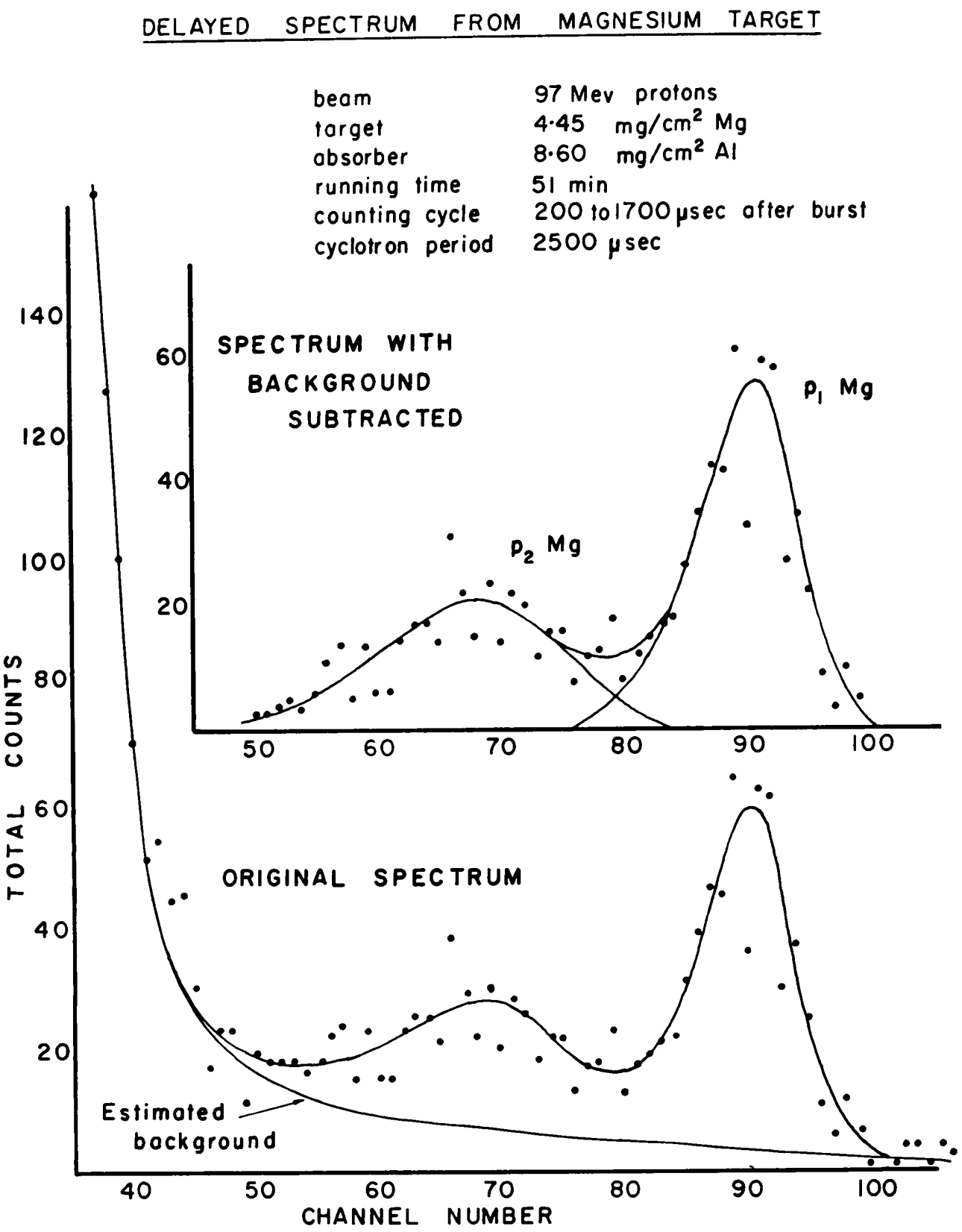

FIG. 5. Spectrum of delayed heavy charged particles from magnesium target.

for both protons and deuterons. Clearly the observed points lie on curves that fall much too slowly for deuterons or any more heavily ionizing particle, while they are well fitted by proton curves. Only two points were obtained for $p_{1} \mathrm{Al}$ because the highest-energy point was too close to the range of the detector to be reliable: no data for this peak are plotted. The data are consistent with $p_{1} \mathrm{Al}$ being due to protons.

It remains to show that these delayed protons are from the bombardment of aluminum and not from some impurity, e.g., the oxygen in the oxide coating on the aluminum foil. This is most convincingly shown by the fact that no other target yielded a very much higher counting rate, including a target of oxygen-containing air.

$\mathrm{Si}^{25}$, a Source of Beta-Delayed Protons

Although only $p_{2} \mathrm{Al}$ and $p_{3} \mathrm{Al}$ have been shown conclusively to be protons, it will be assumed that all the peaks in each of the spectra to be described 


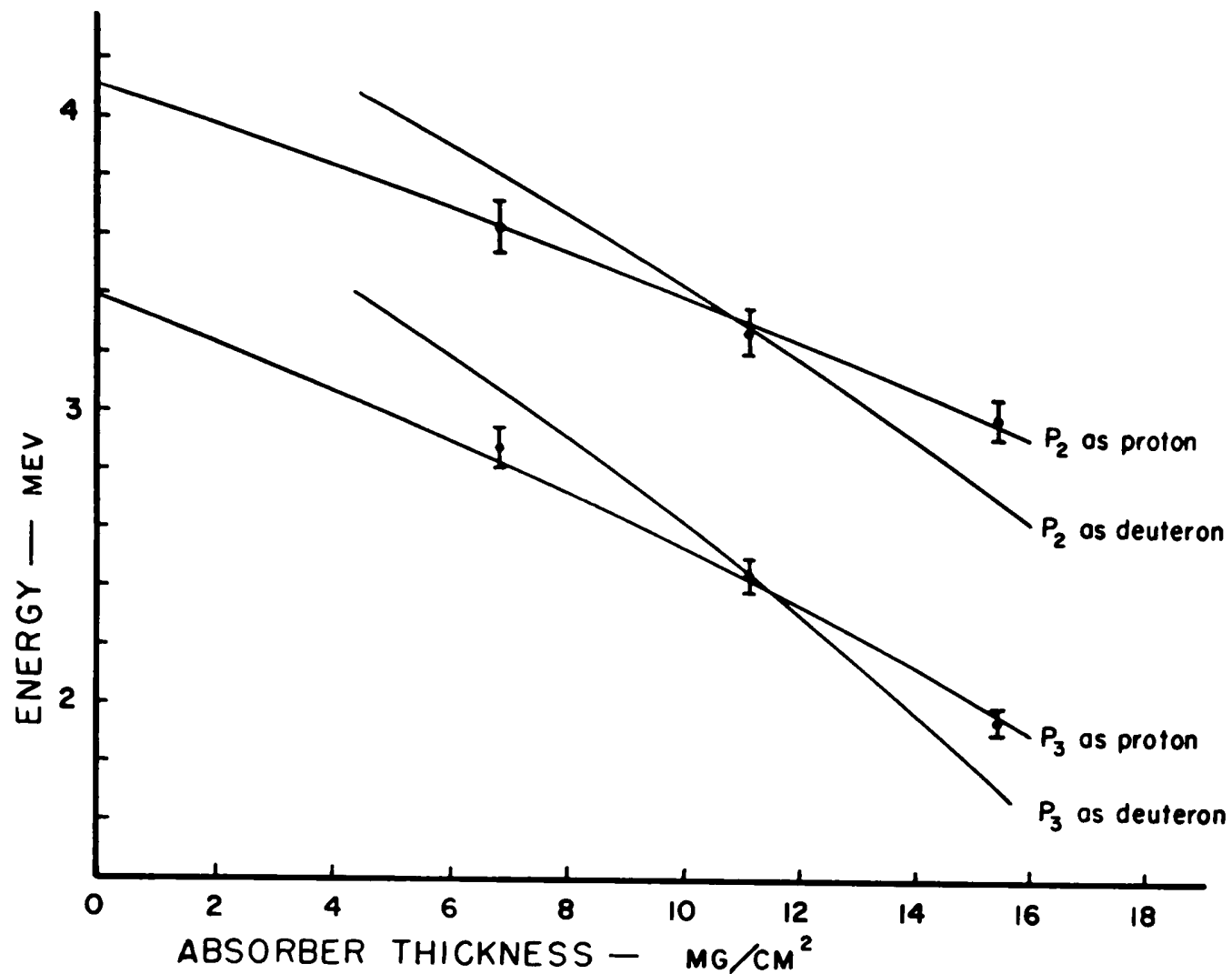

FIG. 6. Residual energy curves showing that the observed peaks are due to protons. The curves are for protons and deuterons of the indicated initial energies. The points represent experimental data.

are due to protons. This is a proper assumption for two reasons. First, as will be seen, all the peaks observed can be accounted for in the same fashion from this assumption. Second, it is hard to see how any other particle can possibly be observed: no neutron-containing delayed particles, except alphas, can be emitted by neutron-deficient light nuclides because the neutron binding energies are as large as the $Q$ values for beta decay; the possibility of observing alphas is eliminated because any alpha that penetrated the $9 \mathrm{mg} / \mathrm{cm}^{2}$ of absorber that was normally over the counter would have to be emitted with at least $12 \mathrm{Mev}$ energy, which again is as large as the $Q$ values for beta decay. Therefore, since some of the peaks have been shown to be protons, since each of the others can be accounted for by assuming that it is due to a proton, and since it is highly doubtful that any other type of delayed radiation could be observed, it will be assumed that all the peaks are due to protons.

Various targets were bombarded and the delayed spectrum recorded in each case. All of these spectra are very similar to that for Al. In all cases, statistics were very poor with consequent large uncertainties in energy and cross section. Table I gives the measured properties of all the intense lines observed. The energy values have been corrected for target, absorber, and counter window thicknesses. Table I also gives cross sections for the production of the proton peaks; usually these turn out to be a few microbarns, but less than one microbarn for an air target.

Having established that delayed protons were emitted following the bombardment of aluminum, an attempt was made to find what type of delayed 
TABLE I

\begin{tabular}{clcccc}
\hline Target & $\begin{array}{c}\text { Energy } \\
\text { of line, } \\
\text { Mev }\end{array}$ & Designation & $\begin{array}{c}\text { Molecular } \\
\text { cross section, } \\
\mu \mathrm{b}\end{array}$ & $\begin{array}{c}\text { Threshold } \\
\text { energy, } \\
\text { Mev }\end{array}$ & $\begin{array}{c}\text { Half-life, } \\
\text { sec }\end{array}$ \\
\hline $\mathrm{SiO}_{2}$ & $4.65 \pm 0.1$ & $p_{1} \mathrm{Si}$ & $4.6 \pm 0.3$ & & $0.3_{-0.1}^{+0.2}$ \\
& $4.17 \pm 0.1$ & $p_{2} \mathrm{Si}$ & $16 \pm 1$ & \\
& $3.39 \pm 0.1$ & $p_{3} \mathrm{Si}$ & $6.2 \pm 0.4$ & & $0.3_{-0.1}^{+0.2}$ \\
$\mathrm{Al}$ & $4.55 \pm 0.1$ & $p_{1} \mathrm{Al}$ & $1.4 \pm 0.1$ & & $0.3_{-0.1}^{+0.4}$ \\
& $4.10 \pm 0.1$ & $p_{2} \mathrm{Al}$ & $3.3 \pm 0.2$ & & \\
& $3.39 \pm 0.2$ & $p_{3} \mathrm{Al}$ & $1.6 \pm 0.1$ & & \\
$\mathrm{Mg}$ & $4.71 \pm 0.1$ & $p_{1} \mathrm{Mg}$ & $3.0 \pm 0.2$ & & \\
& $4.02 \pm 0.1$ & $p_{2} \mathrm{Mg}$ & $2.0 \pm 0.1$ & & \\
$\mathrm{LiF}$ & $4.68 \pm 0.1$ & $p_{1} \mathrm{~F}$ & $7.0 \pm 0.4$ & & \\
& $3.6 \pm 0.1$ & $p_{2} \mathrm{~F}$ & & & \\
$\mathrm{NaF}$ & 4.5 (broad) & $p_{1} \mathrm{NaF}$ & $10 \pm 1$ & & \\
& 3.7 (broad) & $p_{2} \mathrm{NaF}$ & $4.3 \pm 0.2$ & & \\
$\mathrm{~N}_{4.5} \mathrm{O}$ & $4.50 \pm 0.1$ & $p_{1} \mathrm{Air}$ & $0.14 \pm 0.02$ & & \\
$(\mathrm{air})$ & $3.97 \pm 0.1$ & $p_{2} \mathrm{Air}$ & $0.07 \pm 0.02$ & & \\
\hline
\end{tabular}

proton emission was taking place and what the parent nuclides responsible for the delays were. Accordingly, targets of magnesium and silica $\left(\mathrm{SiO}_{2}\right)$ were bombarded. The cross sections for oxygen (air target) are so small compared to those for silica (see Table I) that one may assume that all the silica activities are from the bombardment of silicon. Now, $p_{1} \mathrm{Al}, p_{2} \mathrm{Al}$, and $p_{3} \mathrm{Al}$ each has an analogous peak of the same energy within errors in the spectrum from the silicon target; furthermore, the cross sections in the case of silicon have about the same relative values as those for the case of the aluminum target. It is, therefore, highly probable that these two spectra are of the same set of radiations from the same parent nuclide produced in different ways.

In the spectrum from the magnesium target, Fig. $5, p_{3}$ is entirely missing and the relative intensities of $p_{1}$ and $p_{2}$ are reversed. Clearly, then, the lines from aluminum and silicon are being produced only weakly, if at all, in the bombardment of magnesium. Recalling that in a proton cyclotron the projectile has one unit of charge, we may assume that corresponding lines from the aluminum and silicon targets have common parent nuclides that are isotopes of silicon; it is then impossible to produce these lines in a bombardment of magnesium.

The activation curves shown in Fig. 7 were obtained by bombarding silicon at the various energies indicated on the graphs. Degrading and collimating of the beam was done as previously explained. The semilogarithmic plot in Fig. 7 shows that the three proton lines have the same parent nuclide, for the curves are vertically separated by constant distances and hence are in constant ratios to one another. The linear plot shows that the three lines have the same apparent threshold at about $50 \mathrm{Mev}$. The threshold for the reaction $\mathrm{Si}^{28}(p, p 2 n) \mathrm{Si}^{26}$ can be calculated from known $\beta$-decay $Q$ values (AjzenbergSelove and Lauritsen 1959) and is $31.6 \mathrm{Mev}$ (lab.). Using, in conjunction with this value, estimates of neutron separation energies (Seeger 1961), one finds the threshold for $\mathrm{Si}^{28}(p, p 3 n) \mathrm{Si}^{25}$ to be $49.8 \mathrm{Mev}$ and for $\mathrm{Si}^{28}(p, p 4 n) \mathrm{Si}^{24}$ to 

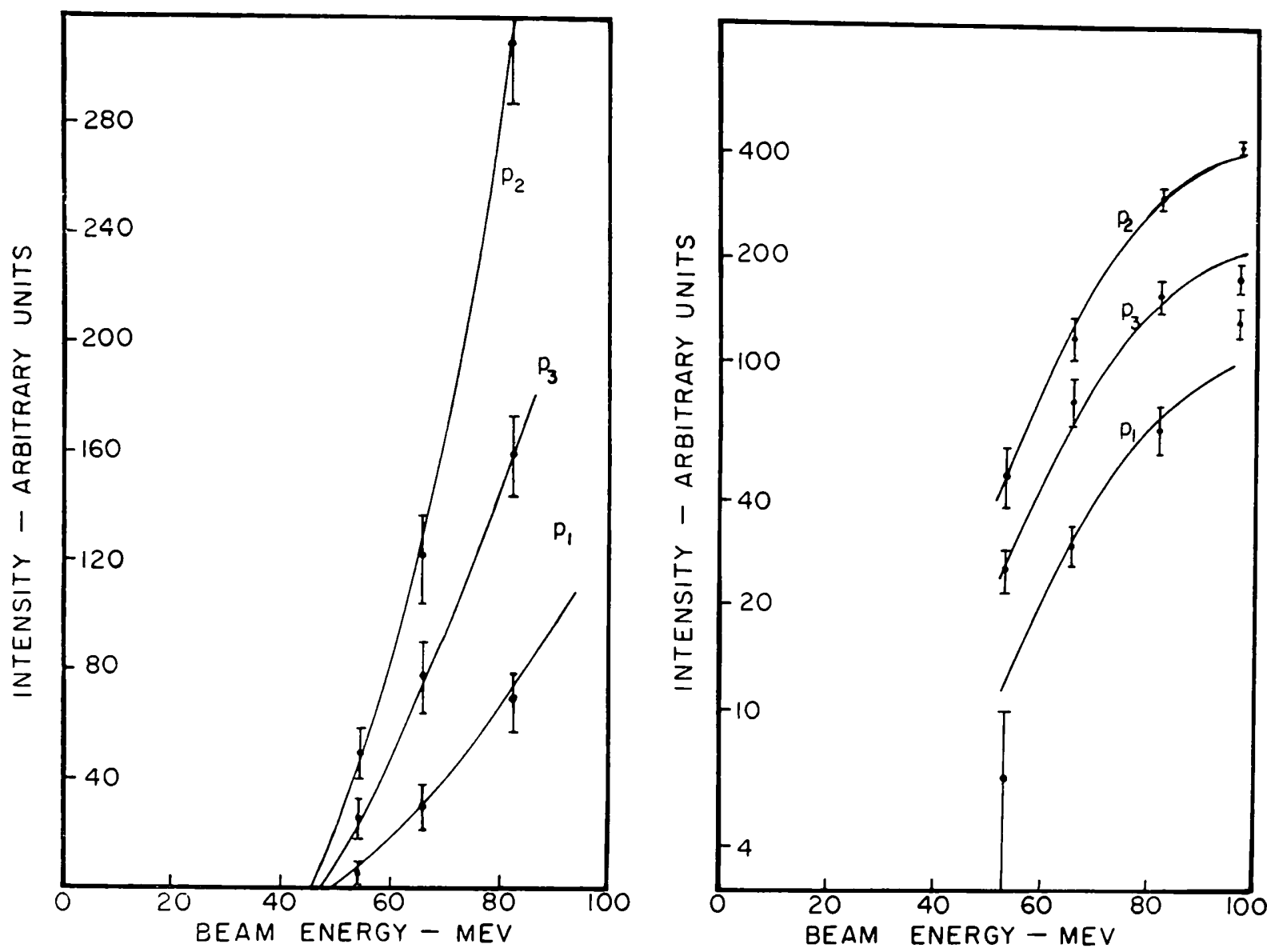

FIG. 7. Activation curves for the three peaks of the spectrum obtained from a Si target. The semilogarithmic plot on the right exhibits the constant ratios between the three energy groups. The linear plot on the left exhibits the common threshold energy at about 50 Mev.

be $64.7 \mathrm{Mev}$. Because of the shape of the curves near threshold and because of the close agreement between the measured thresholds and that calculated for producing $\mathrm{Si}^{25}$, we conclude that $\mathrm{Si}^{25}$ is the parent nuclide for all three proton lines. This conclusion is reinforced by noting that a less complete activation curve for production of the same protons from an aluminum target showed a threshold below $40 \mathrm{Mev}$, consistent with $\mathrm{Al}^{27}(p, 3 n) \mathrm{Si}^{25}$, which has an estimated threshold of $37.8 \mathrm{Mev}$.

Further, the lifetimes of the three lines from the Si target were measured. This was done by gating the cyclotron on for 1 second and off for 1 second. During the off period the pulse-height spectrum was accumulated for consecutive $\frac{1}{4}$-second periods in three of the quarters of the kicksorter's memory, producing a three-point decay curve of the whole spectrum. Figure 8 shows the results obtained for each peak. The lines were drawn parallel with a slope corresponding to a $T_{1 / 2}$ of $0.3 \mathrm{sec}$. This fit is evidently consistent with the data. The equality of these lifetimes is further confirmation that the three peaks are products of the decay of a single nuclide.

The nuclide $\mathrm{Si}^{25}$ has 14 protons and 11 neutrons. The nuclides $\mathrm{Mg}^{25}$ and $\mathrm{Al}^{25}$ are known to be deformed with $\delta=0.3$ approximately (Mottelson and Nilsson 1959). Assuming that $\mathrm{Si}^{25}$ is similarly deformed, the Nilsson diagram predicts $3 / 2^{+}$for its ground state. On the other hand the ground state of its mirror nucleus, $\mathrm{Na}^{25}$, has been assigned as $5 / 2^{+}$(Endt and van der Leun 1962). One 


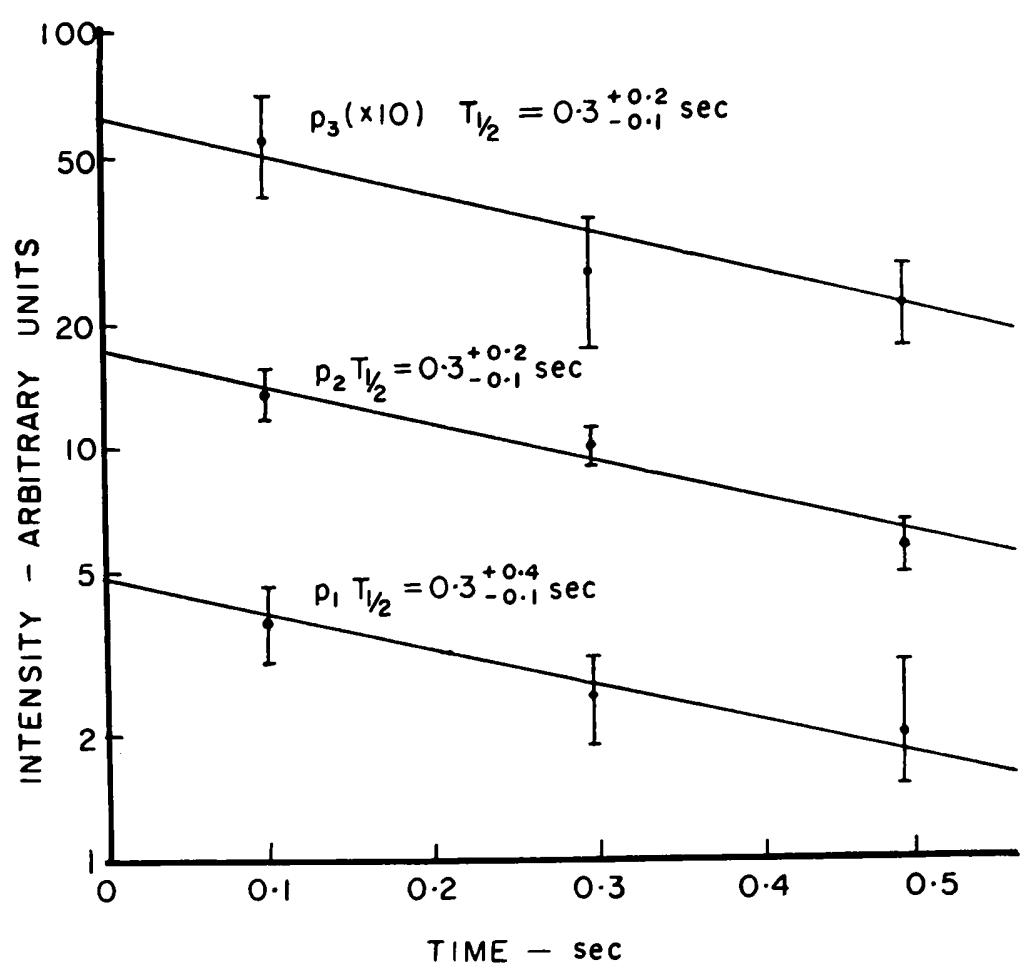

FIG. 8. Decay curves of the three peaks from a silicon target, exhibiting the common halflife of about 0.3 sec. This, therefore, is the half-life of $\mathrm{Si}^{25}$.

may, therefore, assume that the spin-parity of the $\mathrm{Si}^{25}$ ground state is either $3 / 2^{+}$or $5 / 2^{+}$. Hence $\mathrm{Si}^{25}$ decays by an allowed beta transition to the ground state of $\mathrm{Al}^{25}$ and the lifetime can be estimated accordingly. Using Seeger (1961) to obtain an estimate of $10.3 \mathrm{Nev}$ for the $Q$ value, and assuming a $\log f t$ of about 5 , one obtains a half-life of the order of $1 \mathrm{sec}$, in agreement with this experiment. Since the lifetime of a self-delayed 3-Mev proton from Si cannot possibly be as large as $0.3 \mathrm{sec}$, since $\mathrm{Si}^{25}$ is certainly stable against particle emission, and since the observed lifetime of $\mathrm{Si}^{25}$ agrees with that calculated from the theory of beta decay, we conclude that the observed proton lines are beta-delayed protons emitted from excited states of $\mathrm{Al}^{25}$ following the beta decay of $\mathrm{Si}^{25}$.

Figure 9 shows the proposed decay scheme. The peak $p_{2} \mathrm{Si}$, the most intense one, is attributed to the decay of the $6.70-\mathrm{Mev}$ state of $\mathrm{Al}^{25}$ to the ground state of $\mathrm{Mgg}^{24}$. This transition is labeled (2). A proton line at $2.92 \mathrm{Mev}$ due to the decay of this level to the first excited state of $\mathrm{Xg}^{24}$ should also occur, but after being degraded in energy by $10 \mathrm{mg} / \mathrm{cm}^{2}$ of material it would have been hidden under the high background just below 2 Mev and hence not observed. The peaks $p_{1} \mathrm{Si}$ and $p_{3} \mathrm{Si}$ are attributed to the decay of the 7.14 - Mev level of $\mathrm{Al}^{25}$ to the $0^{+}$ground state and the $2^{+}$first excited state of $\mathrm{Mg}^{24}$. The observed intensities of $p_{1} \mathrm{Si}$ and $p_{3} \mathrm{Si}$ are about equal, consistent with the relative energies and spin-changes of these two branches. The intensity of $p_{2}$ is somewhat greater than the sum of those of $p_{1}$ and $p_{3}$, consistent with the slightly greater transition energy for beta decay to the $6.70-\mathrm{N}$ ev level of $\mathrm{Al}^{25}$. Finally, since no level of $\mathrm{Al}^{25}$ in the energy range of interest is known to have a spin and parity appropriate to an allowed beta transition from $\mathrm{Si}^{25}$, this proposed decay scheme does not ignore any expected lines. Thus the proton spectrum from the $\mathrm{Al}$ and $\mathrm{Si}$ targets is accounted for. 


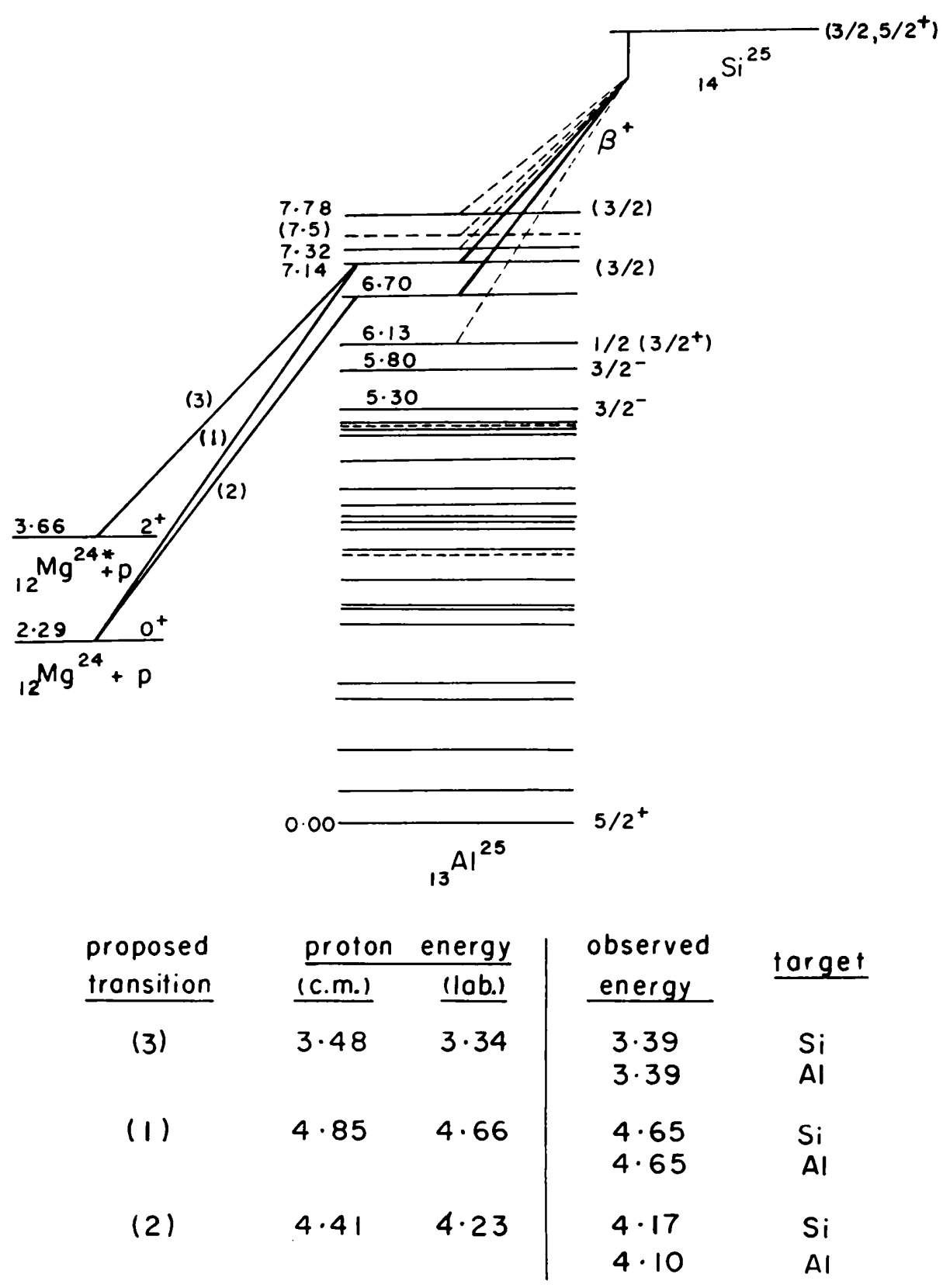

FIG. 9. Proposed decay scheme of $\mathrm{Si}^{25}$ and a comparison of predicted and observed energies. The dashed lines indicate transitions not ruled out by energy or known spin-parity considerations but not leading to observed proton lines.

In summary, it is concluded that all the observed peaks are due to delayed protons and that the spectra from $\mathrm{Al}$ and $\mathrm{Si}$ targets are due to the same radiations. It is concluded from activation and lifetime evidence that these protons are beta-delayed protons emitted from excited states of $\mathrm{Al}^{25}$ following the beta decay of $\mathrm{Si}^{25}$. A decay scheme is proposed that accounts for the observed spectrum and indicates that the 6.70 - and $7.14-\mathrm{Mev}$ levels of $\mathrm{Al}^{25}$ have positive parity.

Intense Lines Due to $(2 k+2,2 k-1)$ Nuclides

The nuclide $\mathrm{Si}^{25}$ is in a very favorable position to be parent to delayed protons for two reasons. First, its $(Z, N)$ is of the form $(2 k+2,2 k-1)$ so that it is parent to the following chain of decays:

$$
(2 k+2,2 k-1) \stackrel{\beta^{+}}{\longrightarrow}(2 k+1,2 k)^{*} \stackrel{p}{\longrightarrow}(2 k, 2 k) .
$$


It is well known that nuclei of the form $(2 k+1,2 k)$, one proton larger than an "alpha-particle nucleus" of the form $(2 k, 2 k)$, have exceptionally low proton separation energies. Second, each of the $(2 k+2,2 k-1)$ nuclides up to a $Z$ of 20 inclusive is the heaviest isotope of its element that has a large $Q$ value for beta decay. This combination of low separation energy and large $Q$ value means that $\beta$ transitions to levels that can yield protons in the energy range of interest have a relatively large transition energy. No neighboring nuclide possesses this combination to the same degree and has a favorable production cross section.

Thus one would expect the spectrum from a Si target to be dominated by protons from the decay of $\mathrm{Si}^{25}$ and the spectrum from a $\mathrm{Mg}$ target to be dominated by protons from $\mathrm{Mg}^{21}$ decay, the next lighter $(2 k+2,2 k-1)$ nuclide. From an $\mathrm{Al}$ target one would perhaps expect $\mathrm{Si}^{25}$ and $\mathrm{Mg}^{21}$ lines. However, identifying $\mathrm{Mg}^{21}$ lines with the spectrum from a $\mathrm{Mg}$ target, we have seen that there seems to be little, if any, contribution by this nuclide to the spectrum from an $\mathrm{Al}$ target. So the reaction $\mathrm{Al}^{27}(p, 3 n) \mathrm{Si}^{25}$ seems more probable than the combined reactions $\mathrm{Al}^{27}(p, 2 p 5 n) \mathrm{Mg}^{21}$ and $\mathrm{Al}^{27}(p, \alpha 3 n) \mathrm{Mg}^{21}$. This is perhaps fairly reasonable. One suspects, however, that with better resolution and statistics some contribution from $M^{21}$ due to the $(p, \alpha 3 n)$ reaction might be observable.

In summary, one expects lines from the decay of $(2 k+2,2 k-1)$ nuclides to dominate the delayed proton spectra from targets of all elements up to and including calcium ( $Z$ of 20 ). This is because these nuclides are in a much more favorable position, energetically, to decay to proton-emitting states than are their neighboring nuclides. Furthermore, $(p, 3 n)$ reactions seem to be more probable, in these light elements, than $(p, \alpha 3 n)$ and $(p, 2 p 5 n)$ reactions combined. This leads to the proton spectrum from targets of both element $(2 k+1)$ and element $(2 k+2)$ being dominated by protons in the decay of nuclide $(2 k+2,2 k-1)$. In accordance with this last hypothesis, the decay of $\mathrm{Mg}^{21}$ should account for the spectra obtained from $\mathrm{Mg}$ and $\mathrm{Na}$ targets, the decay of $\mathrm{Ne}^{17}$ for the spectrum from a $\mathrm{F}$ target, and the decay of $\mathrm{O}^{13}$ for the spectrum from $\mathrm{O}$ and $\mathrm{N}$ targets.

Figures 10,11 , and 12 contain all the information relevant to the verification of this prediction. They were constructed in the following way. In the known level scheme of the intermediate nuclide, i.e., of the daughter of the beta decay, the parent nuclide is assigned a ground-state spin and parity as given by the shell model. All possible beta transitions which, on the basis of known level properties and the assumed ground-state properties of the parent, are thought to involve forbidden transitions are ignored. All other possible transitions that can lead to protons in the observed energy range are shown. Those shown by a dashed line do not lead to an observed proton line. The important points are, first, that all the observed proton energies are accounted for in this way, and second, that all transitions thought to be allowed, on the basis of known level properties, lead to an observed proton energy.

In the case of $\mathrm{Mg}^{21}$, the indicated transitions in Fig. 10 clearly account for the observed spectrum from the $\mathrm{Mg}$ target. It will be explained shortly how 

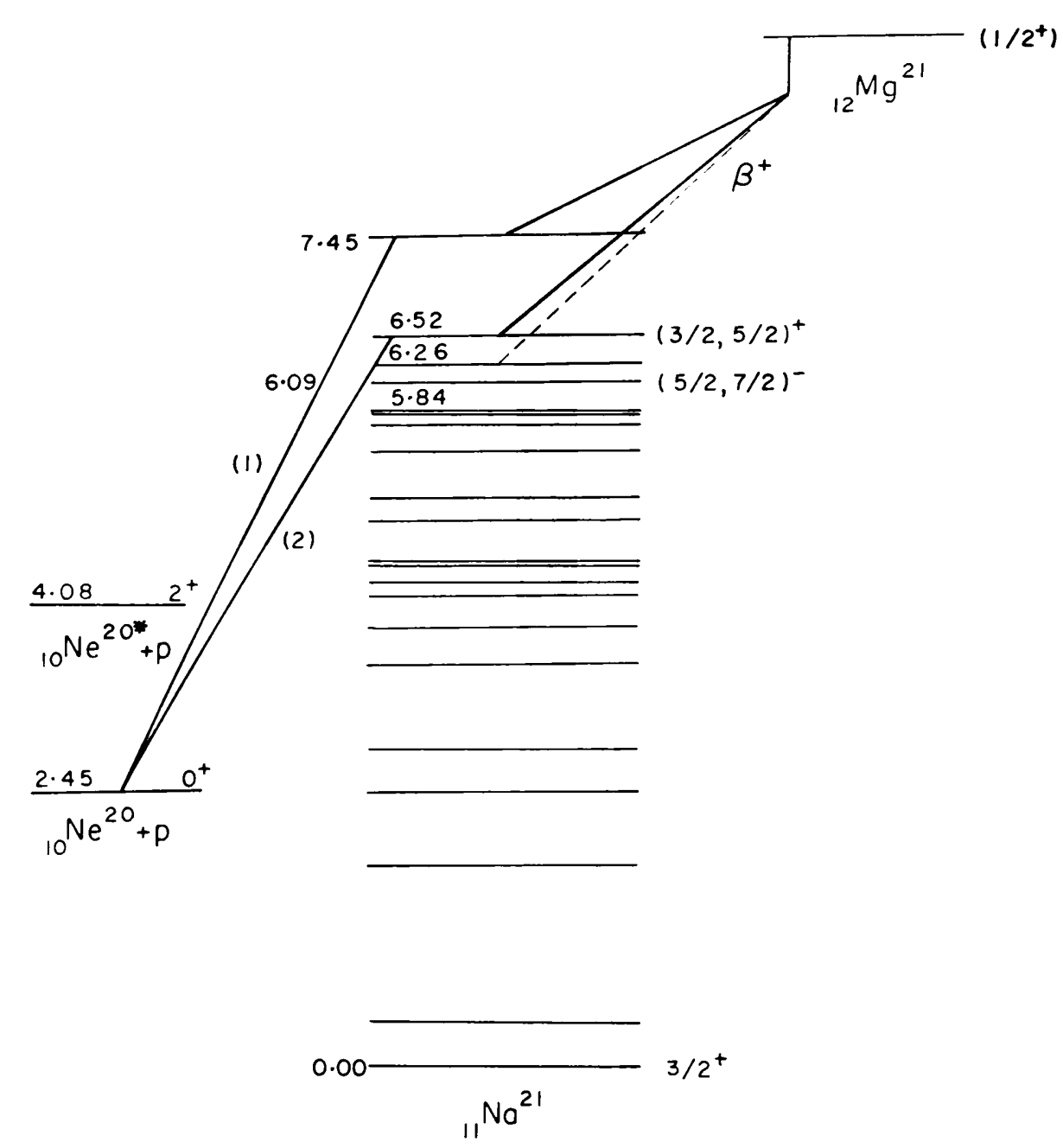

\begin{tabular}{|c|c|c|c|c|}
\hline $\begin{array}{l}\text { proposed } \\
\text { transition }\end{array}$ & $\frac{\text { proton }}{\text { (c.m.) }}$ & $\frac{\text { energy }}{\text { (lab.) }}$ & $\begin{array}{l}\text { observed } \\
\text { energy }\end{array}$ & torget \\
\hline (1.) & $5 \cdot 00$ & $4 \cdot 76$ & $\begin{array}{l}4 \cdot 71 \\
(4 \cdot 5)\end{array}$ & $\begin{array}{l}\mathrm{Mg} \\
\mathrm{NoF}\end{array}$ \\
\hline (2) & $4 \cdot 07$ & 3.87 & $\begin{array}{l}4 \cdot 02 \\
(3 \cdot 7)\end{array}$ & $\begin{array}{l}\mathrm{Mg} \\
\mathrm{NoF}\end{array}$ \\
\hline
\end{tabular}

FIG. 10. Proposed decay scheme from $\mathrm{Mg}^{21}$ and a comparison of predicted and observed energies. The dashed lines indicate transitions not ruled out by energy or known spin-parity considerations but not leading to observed proton lines.

the spectrum resulting from bombardment of $\mathrm{Na}$ in the $\mathrm{NaF}$ target was deduced. It is the same as the Mg target spectrum and hence is also accounted for.

In the case of $\mathrm{Ne}^{17}$ (Fig. 11) there is an initial ambiguity when trying to explain the spectrum from the $\mathrm{LiF}$ target. It is uncertain whether the 5.45or $5.66-\mathrm{Mev}$ level is responsible for the $4.68-\mathrm{Mev}$ observed line. However, the lines in the spectrum from the $\mathrm{NaF}$ target were about $375 \mathrm{kev}$ wide and the system resolution for single proton lines is $250 \mathrm{kev}$. If the spectrum from the $\mathrm{Na}$ part of the target is the same as that from $\mathrm{Mg}$, there is a line at $3.87 \mathrm{Mev}$ from the Na target and a line at $3.6 \mathrm{Mev}$ from the $\mathrm{F}$ target. This accidental doublet accounts for the broad lower-energy peak from the NaF target. Trying to explain the other broad peak in the same way, one must discard the $5.60-\mathrm{Mev}$ 


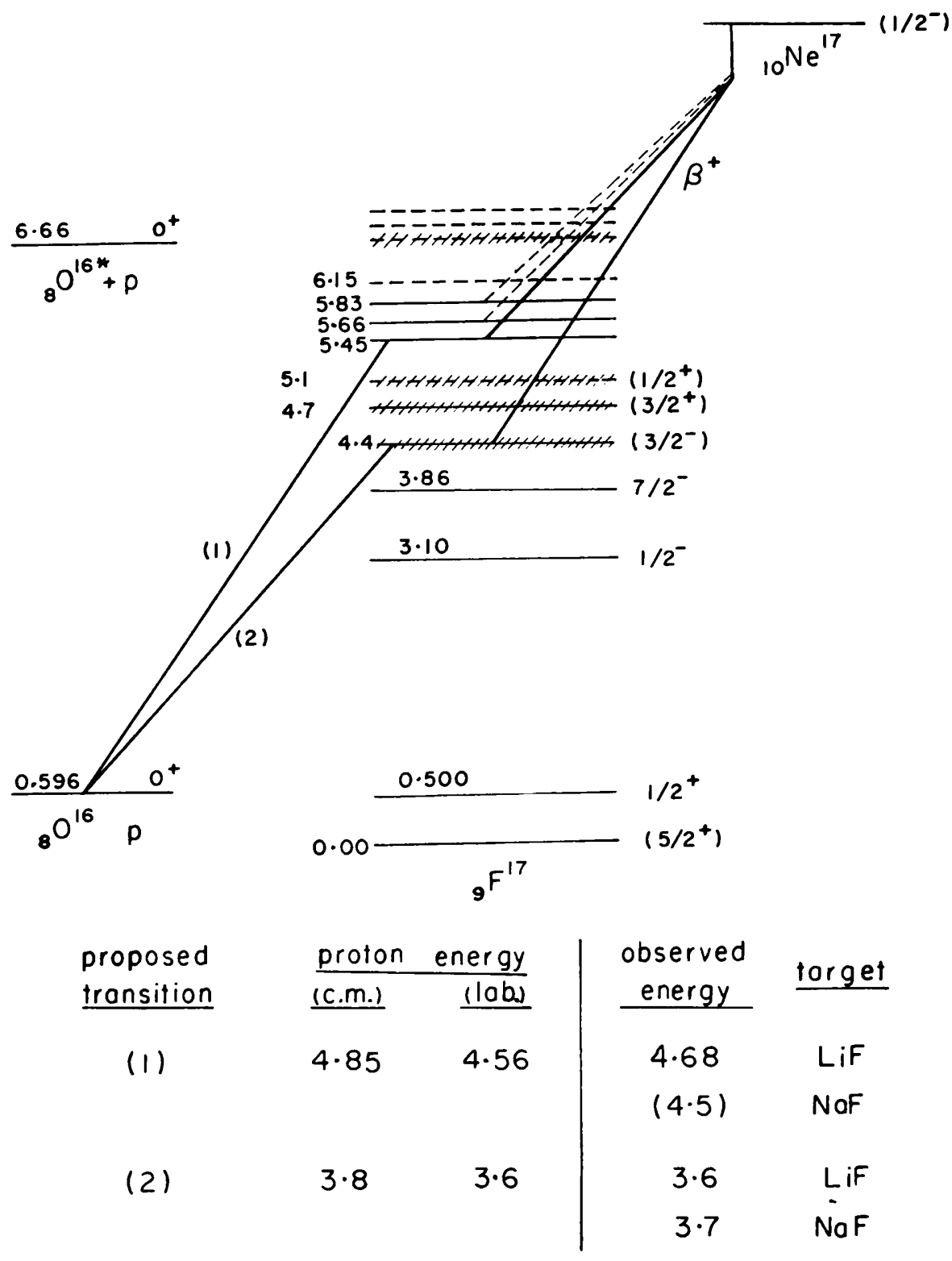

FIG. 11. Proposed decay scheme from $\mathrm{Ne}^{17}$ and a comparison of predicted and observed energies. The dashed lines indicate transitions not ruled out by energy or known spin-parity considerations but not leading to observed proton lines.

level in $\mathrm{Ne}^{17}$ because it leads to protons of the same energy as the higher-energy line from the $\mathrm{Na}$ target. Therefore, by explaining the spectrum from the LiF target and the anomalously broad peaks of the NaF target together, one gets an unambiguous picture of the $\mathrm{Ne}^{17}$ decay and confirms that $\mathrm{Mg}$ and $\mathrm{Na}$ targets both lead to $\mathrm{Mg}^{21}$ decay.

The case of $\mathrm{O}^{13}$ (Fig. 12) is slightly different from the other two cases. To the levels of the beta-decay daughter in the energy range of interest there can be no allowed beta transitions. However, there are possibilities for firstforbidden transitions, and these do in fact account for the observed spectrum from the air target, which contains the elements $\mathrm{N}$ and $\mathrm{O}$. The forbiddenness of these transitions is consistent with the low cross sections observed since they compete with allowed transitions to low-lying levels.

The objection might be raised that these observed lines could come from the decay of nuclides neighboring those mentioned. Perhaps some or all lines from the $F$ target have $F^{15}$ for their source. Since sufficient information on the 


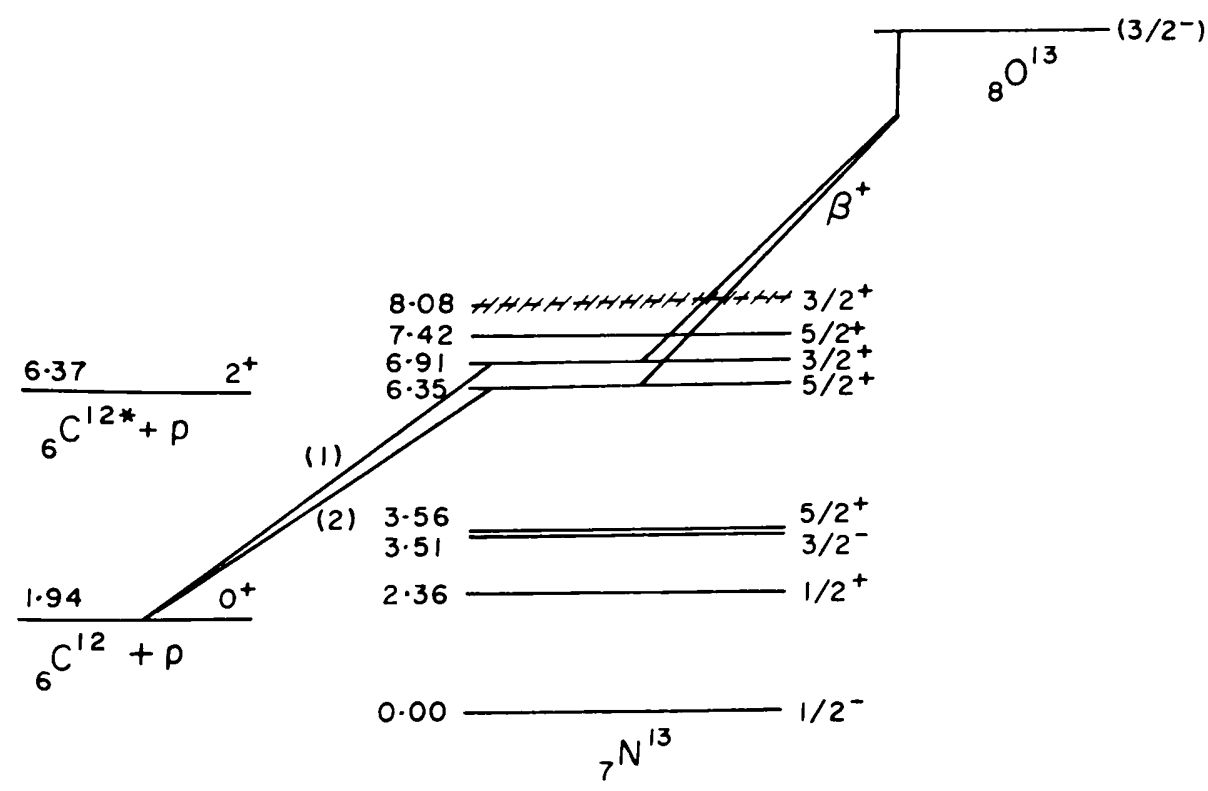

\begin{tabular}{|c|c|c|c|c|}
\hline $\begin{array}{l}\text { proposed } \\
\text { transition }\end{array}$ & proton & energy & $\begin{array}{c}\text { observed } \\
\text { energy }\end{array}$ & target \\
\hline & (c.m.) & (lab.) & & \\
\hline (1) & $4 \cdot 97$ & $4 \cdot 59$ & $4 \cdot 50$ & Air \\
\hline (2) & $4 \cdot 44$ & $4 \cdot 10$ & 3.97 & \\
\hline
\end{tabular}

FIG. 12. Proposed decay scheme from $\mathrm{O}^{13}$ and a comparison of predicted and observed energies. The dashed lines indicate transitions not ruled out by energy or known spin-parity considerations but not leading to observed proton lines.

levels of $\mathrm{O}^{15}$ is not known, one cannot predict the spectrum of this source. However, the important point is that all of the spectrum from a $\mathrm{F}$ target can be explained in a way analogous to the explanations of all the other observed spectra. One line from the air target is at the same energy as a possible line in $\mathrm{N}^{11}$ decay, but the excited state involved lies at $14 \mathrm{Mev}$ energy so the beta decay to it would be extraordinarily highly inhibited. In any case this can only account for part of the observed spectrum from air. One of the lines from the $\mathrm{Mg}$ target can be accounted for by the only expected line from the decay of $\mathrm{Na}^{19}$. Again this cannot account for the whole spectrum. It does not seem likely, on energetic grounds, that the other nuclides neighboring the $(2 k+2,2 k-1)$ nuclides in question emit any delayed protons observable in this experiment.

The information for the construction of Figs. 10, 11, and 12 and for the various associated arguments was taken from Ajzenberg-Selove and Lauritsen (1959), Endt and van der Leun (1962), and Everling et al. (1961).

The hypothesis that the decay of the nuclide $(2 k+2,2 k-1)$ will dominate the delayed proton spectrum from targets of both element $(2 k+2)$ and element $(2 k+1)$ seems to be verified. In particular, by following very reasonable rules for predicting proton lines, all the observed lines are accounted for and all those predictions based on known level properties are borne out. The existence of $\mathrm{Ng}^{21}, \mathrm{Ne}^{17}$, and $\mathrm{O}^{13}$ is assumed since it seems fairly certain that proton lines in the decay of each have been observed. 


\section{Observed Cross Sections}

The cross sections shown in Table I are all of the order of 10 microbarns, except for the air target. The magnitudes of these cross sections support the assignments already made, in the following way. At our bombarding energies, one would expect $(p, 3 n)$ and $(p, p 3 n)$ cross sections to be of the order of 10 millibarns. For concreteness, consider the case of $\mathrm{Si}^{25}$. The estimated $Q_{\beta}{ }^{+}$ is $10.3 \mathrm{Mev}$ to the ground state of $\mathrm{Al}^{25}$, and $3.6 \mathrm{Mev}$ to the $6.70 \mathrm{-Mev}$ state. The corresponding end-point energies are 9.3 and $2.6 \mathrm{Mev}$ respectively. On a fifth-power variation of $\beta$-transition probability, the transition to the upper state will be $1.7 \times 10^{-3}$ as intense as that to the ground state. Since other transitions are also competing, we may guess roughly that the overall branching ratio to the $6.70-\mathrm{Mev}$ state is around $10^{-3}$. Thus the anticipated cross section for delayed protons from this level is around 10 microbarns, as observed. The other cases are similar, except for the air target where extra forbiddenness leads to lower cross sections.

\section{SUMMARY}

It has been shown that delayed proton energy groups are emitted from an aluminum target following bombardment with $97-\mathrm{Mev}$ protons. The delay is due to the $0.3-\mathrm{sec}$ half-life of the previously unreported nuclide $\mathrm{Si}^{25}$ which $\beta^{+}$ decays to proton-unstable excited states of $\mathrm{Al}^{25}$. In the energy range from 2 to $5 \mathrm{Mev}$ the dominant proton lines in all the spectra obtained were ascribed to the decay of $(2 k+2,2 k-1)$ nuclides. Thus protons following $\beta^{+}$decay of $\mathrm{O}^{13}, \mathrm{Ne}^{17}, \mathrm{Mg}^{21}$, and $\mathrm{Si}^{25}$ were found following bombardment of the element of each nuclide as well as the element of one-lower atomic number. One would expect this pattern to continue up to calcium. Thus one expects $\mathrm{S}^{29}, \mathrm{Ar}^{33}$, and $\mathrm{Ca}^{37}$ to be observable via delayed proton emission.

The material presented here does little more than demonstrate the existence of delayed proton radioactivity. It seems obvious that with greater beam intensity and better energy resolution, both easily obtainable, it will be possible to observe indirectly the ground-state $\beta^{+}$decay of many new protonrich nuclides. New information about proton-emitting excited states of the nuclides involved should also be forthcoming. It would be particularly interesting to observe the delayed proton spectra at lower energies than was attempted here (below $2.5 \mathrm{Mev}$ ). One would expect proton lines at these energies to be more intense and perhaps more numerous.

\section{ACKNOWLEDGMENTS}

Two of the authors (R. B. and R. McP.) would like to thank the National Research Council of Canada for Studentships held during the course of this work. This work was supported by a grant from the Atomic Energy Control Board.

Note ADDED IN PROOF: When this article was in press, we received the translation UCRL-TRANS-919 (1963) of an unpublished report JINR (Dubna) P-1072 (1962) by V. A. Karnaukhov, G. N. Ter-Akopyan, and V. G. Subbotin. 
These workers searched for particles emitted from a foil acting as a catcher for products recoiling from thin targets bombarded with $130-\mathrm{Mev} \mathrm{Ne}^{20}$ ions. The detectors were $E$ and $d E / d x$ gas counters. With targets of both nickel and tantalum, they observed a few events due to particles with energies between 3 and $4.5 \mathrm{Mev}$, delayed by at least $0.1 \mathrm{sec}$, and much less ionizing than $\alpha$ particles. The authors attribute their results to delayed protons; no further information is given in their report.

\section{REFERENCES}

Ajzenberg-Selove, F. and Lauritzen, T. 1959. Nucl. Phys. 11, 1.

BARTON, R. 1963. Ph.D. Thesis, McGill University, Montreal.

Barton, R. and McPherson, R. 1963a. Bull. Am. Phys. Soc., Ser. II, 8, 357.

1963b. Phys. in Can. 19, No. 3, 37.

Booth, E. T., Duning, J. R., and Slack, F. G. 1939. Phys. Rev. 55, 876

ExDT, P. M. and VAN DER LeUn, C. 1962. Nucl. Phys. 34, 1.

Everling, F., Koenig, L. A., Mattacch, J. H. E., and IIApstra, A. H. 1961.1960 Nucl. Data Tables, U.S. At. Energy Comm.

Goldaxsky, V. I. 1960. Nucl. Phys. 19, 482.

1961. Nucl. Phys. 27, 648.

Le Couteur, K. J. 195. . Proc. Roy. Soc. (London), Ser. A, 232, 236.

McPherson, R. and Barton, R. 1963. Phys. in Can. 19, No. 3, 36.

Moore, R. B. 1962. Ph.D. Thesis, McGill Tniversity, Montreal.

Mottelson, B. and Nilsson, S. G. 1959. Kgl. Danske Videnskab. Selskab, Mat. Fys.
Skrifter, 1, No. 8.

Pappas, A. C. and Ridstam, G. 1960. Nucl. Phys. 21, 353.

Roberts, R., Meyer, R. D., and Waig, P. 1939. Phys. Rev. 55, 876.

Seeger, P. A. 1961. Nucl.' Phys. 25, 1. 


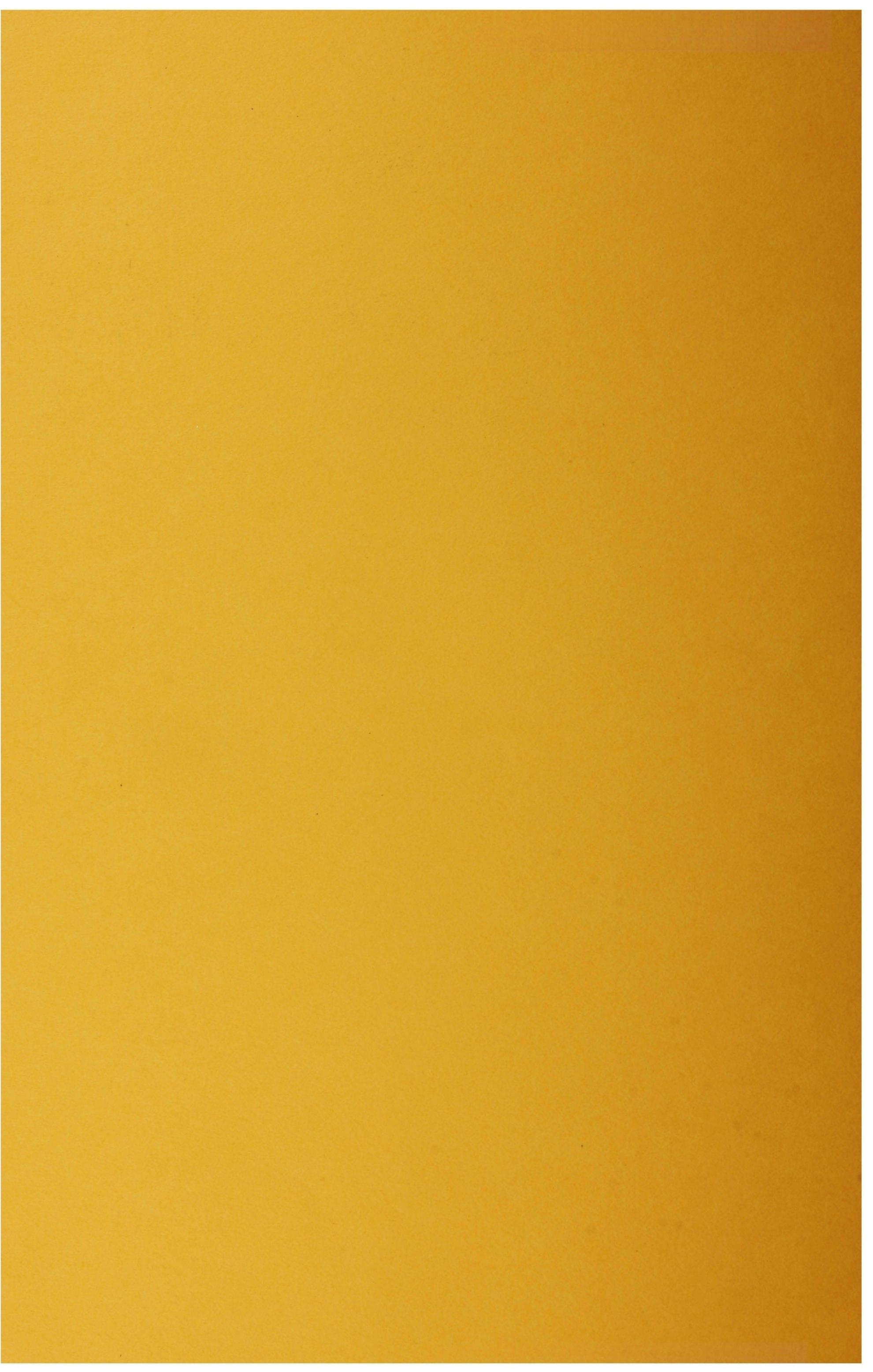

